\title{
CLINICAL OBSERVATIONS WITH THE SLIT-LAMP
}

\author{
BY \\ L. WEBSTER FOX, M.D. \\ PHILADELPHIA
}

THE popularity of the slit-lamp in ophthalmic examinations has led enthusiastic clinicians to claim more virtues for the instrument than can always be substantiated. In routine examinations it is likely to be disappointing, but in selected cases there is no question of its importance. But, even here, the information, while frequently interesting, will be of no more value in guiding the treatment than the more commonplace instruments and methods. Other observers have also stressed this feature. The following cases well illustrate the value and some of the limitations of this type of ophthalmic examination.

Case 1.-This patient was a young man, born in America of Yiddish-Polish parentage who came to this country from Warsaw thirteen years before. The father and mother were first cousins. One brother, now deceased, was born deaf and dumb. The patient presented features that I am inclined to regard as in some way connected with this consanguinity of parentage.

Looking into the eyes with the pupils dilated and employing the ordinary oblique illumination and condensing lens, a stellate lenticular opacity was seen in each eye. With the ophthalmoscope with a high convex lens, these opacities assumed a more robust character. In the right eye the stellate opacity seemed to occupy the entire posterior pole of the lens and resembled a piece of coral having six main branches or arms which subdivided again and again as the periphery was reached. These arms had thickness and were roughened as is the case with coral. They were not flat, although the picture with the ophthalmoscope did not show more than two dimensions. In the left eye there were eight branches. This type of opacity. I have liked to describe as "moss agate." The left eye showed lamellated changes in the vertical meridian of the lens, more or less angularly situated, which presented the reflexes of diffraction. The fundus showed atrophic changes in the retina, the nerves and the vessels together with stellate pigment deposits in the periphery of the fundus-a retinitis pigmentosa in which the pigment was not profuse, but in which the atrophic changes were decidedly pronounced.

Upon examination with the slit-lamp the features of this case became greatly amplified. In the right eye the opacity in the lens became definitely located in the posterior capsule. The 
aqueous beam showed the presence of debris. The stellate figure in the posterior capsule showed some thickness to its structure, and was yellowish brown in colour with light-reflecting surfaces. With the ophthalmoscope it was black and the thickness had to be assumed. In the left eye the posterior opacity was not quite so dense, but the lens substance showed the vertical lamellae rather angularly placed, which gave mother-of-pearl reflexes. It is difficult to state whether the slit-lamp added much in the diagnosis over the other methods of examination, but certain it is, it considerably amplified the information obtained. The status of the case could have been determined without it, although it provided an extremely good case for clinical denonstration.

Case 2.-This patient was the subject of trachoma in the right eye with pronounced pannus. A peridectomy had been performed and the minute examination of the cornea was deemed advisable to note the effect. The iris incidentally was found to be reacting normally to light stimuli and there were no adhesions. It would have been impossible to determine this with the ordinary methods of examination. The intense illumination and the corneal microscope demonstrated this. The vascularity of the cornea was readily demonstrated and all the spaces were found to be filled in with inflammatory exudate. Empty vessels were also to be seen. The involution of the corneal inflammation could be determined in places, thus confirming the gross, improvement noted by the patient. The microscopy of the corney afforded by this means of examination is truly wonderful.

Case 3.- Here the ieft eye had been the subject of a remote penetrating injury involving the cornea and iris. There was complete obliteration of the pupil with complete posterior synechiae. Part of the iris was incarcerated in the corneal scar above. With the slit-lamp the cornea showed two superficial vertical scars with a highly refracted elliptical area in the deeper layers, suggesting a deep vacuolization of the cornea. The cornea also showed evidence of degeneration. The vessels in the upper part of the cornea extending to the iris produced a very interesting picture. In this case the slit-lamp only added detail to the obvious.

Case 4.--In this case in both eyes there had been remote interstitial keratitis. The patient had been under observation since early childhood and had progressed towards resolution after most intensive treatment. He was now apparently in very good condition. Hutchinson teeth could be demonstrated. The slitlamp showed in the right eye that the cornea was still quite hazy and the seat of small curved deposits and some pigment. Posterior 
synechiae were demonstrated below and a fibrous cloudiness of the anterior capsule could be made out. Many empty and many partially filled vessels could be made out in the cornea. The demonstration of these vessels and nerves in the cornea by means of the slit-lamp is one of the most interesting features in the use of this instrument.

C'ase 5.-In the left eye, vessels could be made out in the cornea without any trouble. Pigment was present in the anterior capsule, which was quite rough and appeared to be organized. A small, round, yellow floating body suggesting at times a posterior polar cataract, and behaving very much like a jelly fish in its migrations, but doubtless a bit of organized lymph, could be seen moving about with the grace of a descending parachute. This appeared golden yellow, like a ball of glowing metal at times, and gave the entire picture a most unusual appearance.

Case 6.-Remote iritis. This case showed the changes in the crypts and vessels of the iris in the usual manner. The picture shown by the slit-lamp in cases of this character is very interesting but it must be confessed that up to the present the knowledge gained thereby has not influenced the practical side very much.

Case 7.-Posterior polar cataract in both eyes. Lenticular changes always present interesting features with this form of examination, and it is always with great difficulty that we can leave them for others of more practical importance. The history stated that the trouble with the eyes had existed from birth. In the right eye there was a central corneal opacity, with wrinkling of the anterior capsule immediately behind the central corneal opacity. A faint line extended posteriorly from the dot on the anterior capsule to the opacity in the posterior capsule which was considerably larger, resembling a small pearl collar button with its base placed in the posterior capsule and the tip anteriorly. The entire lens was highly refractive.

In the left eye the condition was much the same, including the central corneal opacity and the dot on the anterior capsule of the lens. Those that regard these conditions as originating in a source anterior to the eye will obtain some justification for their views in the findings on the anterior surface in cases of this character.

Case 8.-Boy with irideremia of both eyes. This boy was completely blind. There was no vestige of iris in either eye, and a pronounced glaucoma existed in both eyes. In the right eye there was a marked staphyloma at the corneo-scleral junction-the weakened coats could no longer stand the high 
intraocular tension. Perhaps it was merely coincidental but the lens appeared to be closer to the external coats of the eye above than below. There was no staphyloma below. While the pupillary area was filled with a pinkish reflex there were other features of interest. In both eyes the supporting fibres of the suspensory ligament of the lens, which we have already remarked about at length in the report of a case of ectopia lentis, were shown here to great advantage. The anterior capsule of the lens showed a whitish reflex, and the body of the lens was highly refractive. In the posterior capsule in both eyes there were large stellate figures similar to those observed in the case reported first in this series. This case is progressing rapidly towards incurable blindness, if it has not already reached that point. It presents many problems for careful consideration. The presence of glaucoma in the absence of the iris gives us something to ponder about. Here we have the equivalent of a total iridectomy yet tension is high and the globe threatens to rupture above. The cornea, however, presents none of the characteristics of ordinary buphthalmos. The possibility of benefit from a posterior sclerotomy engages our attention. More extensive surgical interference seems rather hazardous in view of the obvious disintegration of the ocular structures as indicated by the staphylomatous change.

Case 9.-Cyst of the cornea. This case was examined and reported in another connection but the previous examination was made with the pupil in repose. At this time the pupils were dilated by atropin. This youngster had been operated upon about two years previously for the relief of a convergent squint. An advancement had been performed. Three months ago the mother noticed whitish spots beginning to appear on the front of the left eye. The heaping up of the conjunctiva on the site of the suturing of the muscle in its new situation had existed since the time of the operation although appearing more red in colour than at the present time. Beneath and to the corneal side a $\mathrm{V}$-shaped separation of the sclera was visible through which the dark blue of the choroidal coat could be readily seen. As the limbs of this $\mathrm{V}$ approached the cornea they were projected into it as cloudlike broad opacities separated by a more or less transparent area. Vessels entered the opacities above and below. The corneal block produced by the slit-lamp separated into two, the anterior one curving outward and the posterior one curving inward as the illumination passed over the area enclosed by the opacities. This seemed to indicate the presence of a cyst of the cornea communicating with a rupture of the sclera, which was probably the source of the cystic change in the cornea. 